\title{
Variación del nitrógeno fecal de Odocoileus virginianus a diferentes tiempos de exposición ambiental
}

\author{
Salvador Mandujano ${ }^{1^{*}}$
}

Introduction: Successful wildlife management requires reliable indicators of the nutritional status of the animals in order to assess the relationship between them and their habitat. Fecal nitrogen (FN) has often been considered to be a good indicator of diet quality. FN analysis has been applied extensively in the temperate region of the range of the deer Odocoileus; however, little is known about the usefulness of this approach in the tropical habitats where the white-tailed deer O. virginianus is hunted. This study presents data on the variation in FN concentration with increased time of exposure. Specifically, we discuss the implications for field sampling in tropical habitats where environmental conditions can rapidly degrade deposited deer feces.

Methods: Quantification of FN was performed with 18 freshly deposited pellet-groups collected at the end of the dry season of 2008 (April-May) in a protected natural area in Puebla state, Mexico. Samples were transported frozen to a Botanical Garden in Xalapa, Veracruz, where they were kept under controlled environmental conditions. Each pellet-group was sampled every 15 days, up to 60 days, by which time most of the droppings were covered with fungi due to the high humidity at the site. FN concentration was determined according to the micro-Kjeldahl method. An analysis of variance for repeated measures to detect differences in FN considering the deposition time and fecal groups as factors was applied using Im function in R.

Results: FN varied significantly depending on the exposure time of the feces. The initial concentration was 2.29 $\mathrm{mg} / \mathrm{ml}$ and this value remained constant for 15 days, subsequently decreasing to a minimum of $0.019 \mathrm{mg} / \mathrm{ml}$, which was reached 60 days after the start of the experiment.

Discussion and Conclusions: The use of fecal indexes may be a feasible non-invasive method by which to study diet quality, and an alternative to other techniques that imply disturbance, stress, or death of wild ungulates. In addition, fecal index is inexpensive and easy to apply on large sections of wild animal populations, throughout the year. Our results suggest that use of this method can give reliable results relating to white-tailed deer FN for up to two weeks post-defecation. In contrast, the results of another study suggest that feces collected for up to 24 days post-defecation, in September/October in temperate habitat in US, can be used to estimate FN as well as other nutritional characteristics. Seasonal variation in environment conditions and diet composition could explain these differences. We therefore recommend cleaning permanent sample plots and then returning to locate and collect fresh droppings within these plots.

Key words: fecal nitrogen, nutritional quality, Odocoileus virginianus, tropical habitats.

\section{Resumen}

El análisis de nitrógeno fecal (NF) es una técnica alternativa utilizada para la evaluación de la calidad de la dieta del venado cola blanca Odocoileus virginianus. Este artículo presenta los datos de un experimento que evalúa la pérdida NF de 18 grupos fecales bajo condiciones controladas. Los resultados indicaron que los valores de NF se mantuvieron constantes

'Red de Biología y Conservación de Vertebrados, Instituto de Ecología A. C., km 2.5 Camino a Coatepec No. 351, Xalapa 91070, Ver. Mexico. E-mail Salvador.mandujano@inecol.edu.mx (SM)

${ }^{*}$ Corresponding author 
durante un máximo de 15 días y disminuyó de forma exponencial a partir de entonces. Por lo tanto, muestras con más tiempo no son adecuados para la estimación confiable del NF. Se discuten las implicaciones para el muestreo.

Palabras clave: calidad nutricional, hábitats tropicales, nitrógeno fecal, Odocoileus virginianus.

El manejo exitoso de la fauna silvestre requiere indicadores confiables de la situación nutricional de los animales (Robbins 1993). En el caso de los venados y otros rumiantes, un método común es evaluar la calidad de las plantas que consumen; sin embargo, este procedimiento puede subestimar las concentraciones de nitrógeno del material ingerido (Hewitt 2011). Otros enfoques incluyen el análisis de nitrógeno en la sangre y en la orina (Delgiudice et al. 1990) los cuales son muy precisos pero poco prácticos para su uso con ungulados en vida libre. Alternativamente, el nitrógeno fecal (NF) a menudo ha sido considerado como un buen indicador de la calidad de la dieta (Aldezabal et al. 1993; Irwin et al. 1993; Garin et al. 2001; Kamler y Homolka 2005; Sacconi et al. 2007). Aunque algunos estudios han comprobado la relación del NF y algunos parámetros de calidad de la dieta de varias especies de ungulados, en diferentes zonas y estaciones del año, todavía hay algunas dudas sobre su confiabilidad (Thompson 1987). Se ha sugerido que el NF puede reflejar patrones estacionales y espaciales de la calidad de la dieta a nivel poblacional (Ueno et al. 2007; Verheyden et al. 2011).

El análisis del NF se ha aplicado ampliamente en la región templada para manejar a los venados del género Odocoileus (Leslie et al. 1985, 1989; Howery y Pfister 1990; Jenks et al. 1990; Massey et al. 1994; Osborn y Jenks 1998; Osborn y Ginnett 2001). Sin embargo, poco se sabe acerca de la utilidad de este enfoque en los hábitats tropicales (Gallina et al. 2014) donde es ampliamente aprovechado el venado de cola blanca O. virginianus tanto en vida libre como en criaderos intensivos (Gallina et al. 2010; Ortega et al. 2011). Durante los monitoreos poblacionales es común encontrar grupos fecales con distinto tiempo de deposición y generalmente es difícil decir cuántos días se han mantenido en campo después de la defecación (Camargo-Sanabria y Mandujano 2011). Se ha documentado que el NF se pierde tan rápidamente que la estimación del valor nutricional de las plantas consumidas por los herbívoros a partir del análisis del contenido de nitrógeno de muestras fecales viejas es poco preciso (Jenks et al. 1990). Por esta razón, es necesario evaluar las condiciones que afectan a la confiabilidad de los valores NF en áreas específicas. El presente estudio tuvo como objetivo analizar la variación de la concentración de NF conforme aumenta el tiempo de exposición en un hábitat del centro de México. Se discuten algunas implicaciones para el muestreo de campo en hábitats donde las condiciones ambientales pueden degradar rápidamente las heces depositadas por los venados. 


\section{Material y Métodos}

Se colectaron excrementos recién defecados por venados cola blanca mantenidos en cautiverio dentro del área natural protegida "Flor del Bosque" en el estado de Puebla, México. El sitio tiene 614 ha de superficie, con una temperatura media anual de $18{ }^{\circ} \mathrm{C}$ y altitud de 2,200 - 2,470 metros, dominado por bosque templado de encinos y pinos. En el sitio viven 35 venados cola blanca ( 12 machos, 15 hembras y 8 juveniles) dentro de un área cercada de 4 ha (Martinez-Romero 2004). Se colectaron 40 grupos fecales frescos al final de la época seca del 2008 (Abril-Mayo). Las muestras se transportaron en una hielera al Jardín Botánico "Francisco Javier Clavijero" en Xalapa, estado de Veracruz. Este sitio es manejado por el Instituto de Ecología A. C. Está enclavado en los contrafuertes de la Sierra Madre Oriental, a $2.5 \mathrm{~km}$ al suroeste de la ciudad de Xalapa, se localizan tanto el Jardín como el Santuario. Ambas áreas tienen una altitud media de 1,400 msnm, en tierras tropicales de montaña, con clima templado. El sitio además ofrece instalaciones y condiciones para la realización de experimentos permanentes.

Se colocaron las muestras en un sitio protegido de la lluvia directa la cual afecta las muestras al aumentar el grado de humedad y proceso de descomposición de las mismas. Además, se eligió el sitio por cuestiones logísticas para realizar las mediciones periódicas del contenido de nitrógeno. El contenido de NF se cuantificó cada 15 días hasta los 60 días cuando la mayoría de los grupos fecales mostraban signos claros de envejecimiento (Valdespino y Martínez-Romero 2014; Figura 1). En el transcurso del experimento se cubrieron de moho algunas muestras. Por lo que al final los análisis se realizaron con únicamente 18 grupos fecales que no mostraron ningún signo de contaminación. La concentración del NF fue determinada de acuerdo al método micro-Kjeldahl (AOAC 1999).

Para el análisis estadístico se verificó en primer lugar que los datos tuvieran una distribución normal para lo cual se empleó la prueba gráfica Normal Q-Q entre los cuartiles teóricos y los residuales estandarizados (Dalgaard 2008). Comprobado lo anterior, se aplicó un análisis de varianza de medidas repetidas para detectar diferencias en el NF considerando como factores el tiempo de depósito $(0,15,30,45$ y 60 días) y los grupos fecales $(0,1,2 \ldots 18)$ empleando la función Im en R ver. 2.15.3 (R Development Core Team 2012). El modelo empleado fue: $I m$ (formula = nitrógeno_fecal $\sim$ as.factor (días) + as.factor (grupo_fecal)). En caso de valor significativo $(P<0.05)$ se empleó la prueba a posteriori NK para comparación de medias.

\section{Resultados}

La concentración del NF varió significativamente dependiendo del tiempo de deposición de los excrementos (Figura 2; $F=128.26$, g. I. $=4,68, P=0.0001$ ); mientras que no fue significativa respecto al grupo fecal $(F=1.49, \mathrm{~g} . \mathrm{I} .=17,68, P=0.12)$. La concentración de nitrógeno inicial fue de $2.29 \mathrm{mg} / \mathrm{ml}$ y se mantuvo hasta los 15 días (post hoc prueba N-K, $P=$ 0.33); posteriormente disminuyó hasta alcanzar un mínimo de $0.019 \mathrm{mg} / \mathrm{ml}$ a los 60 días de iniciado el experimento $(P=0.0001)$. 


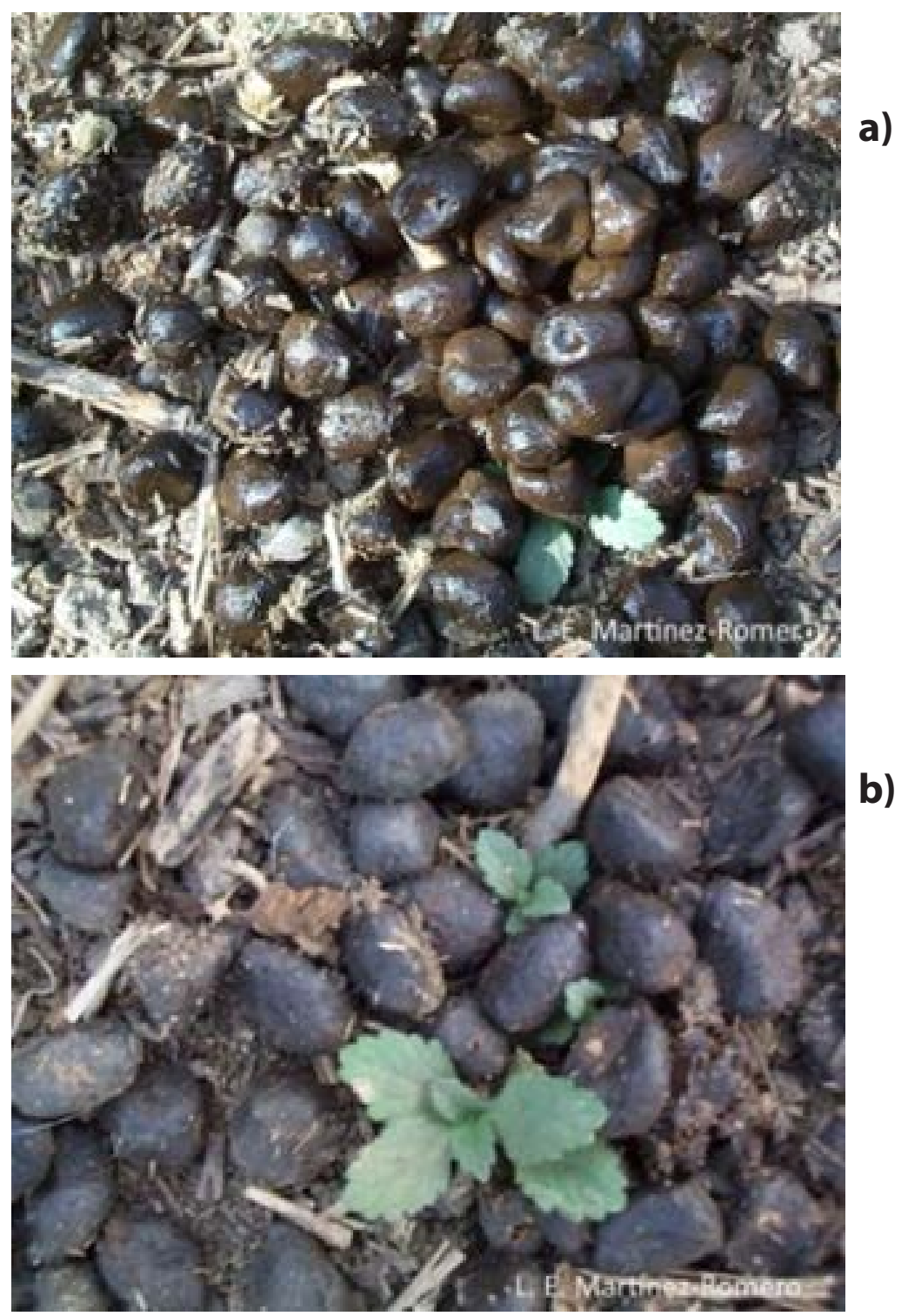

Figura 1. Imágenes mostrando el cambio en coloración y aspecto experimentado por las excretas de venado cola blanca en el momento en que se defecan (a) y varios después (b). Fotografías proporcionadas por L. E. Martínez-Romero.

\section{Discusion}

Los resultados sugieren que el uso de este método puede dar resultados confiables de la concentración de NF en el excremento del venado cola blanca hasta dos semanas después de la defecación. En contraste, en un estudio con esta especie pero en hábitat templado en EE.UU durante septiembre y octubre, se sugiere que las heces recogidas hasta 24 días después de la defecación pueden ser utilizadas para estimar NFy otros aspectos nutricionales (Jenks et al. 1990). La variación estacional de las condiciones del hábitat y la composición de la dieta podrían explicar estas diferencias (Garin et al. 2001). El venado cola blanca habita diferentes tipos de vegetación en la región tropical, pero principalmente en el bosque seco tropical, sabanas y vegetación secundaria (Gallina et al. 2010). A diferencia de las latitudes del Norte donde los inviernos son severos, en zonas tropicales los venados enfrentan una humedad relativamente alta durante la estación lluviosa y la incidencia solar alta durante la estación seca (Mandujano 2010). Esto provoca la rápida degradación de las heces que están 
expuestos directamente al ambiente debido a la actividad de los microorganismos, hongos e insectos, o un secado rápido durante la temporada seca (Valdespino et al. 2007).

Figura 2. Perfil del nitrógeno fecal (mediana \pm cuartiles) a través del tiempo para el venado cola blanca.

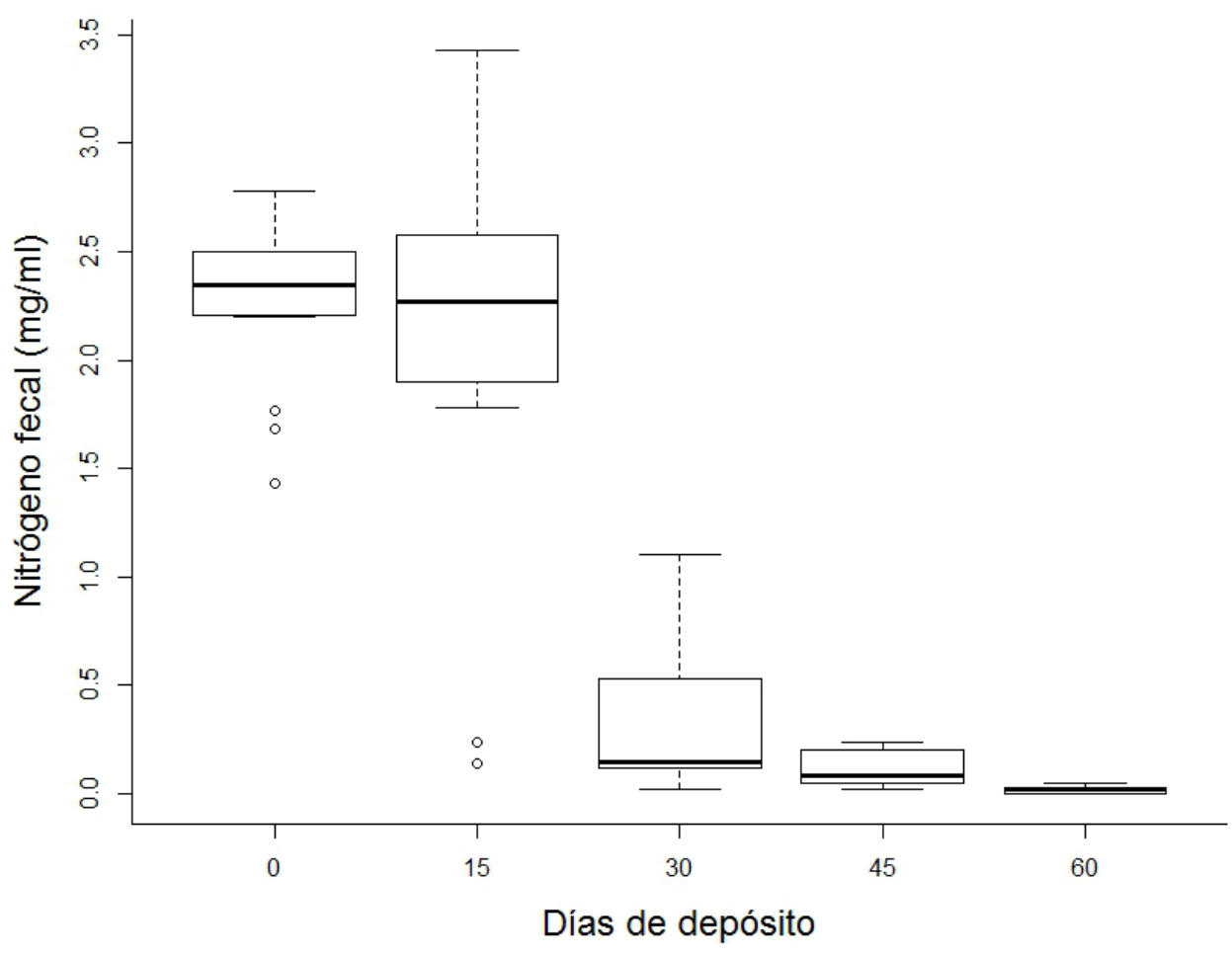

Las bajas densidades poblacionales de esta especie en muchas localidades tropicales, hacen que sea difícil encontrar grupos fecales frescos (color oscuro, brillante y con mucosidad, ver Figura 1), e incluso no es sencillo encontrar excremento con menos de 14 días de depósito (Garin et al. 2001; Martinez-Romero 2004). Por lo tanto, se recomienda limpiar parcelas permanentes de muestreo y revisar nuevamente antes de dos semanas para recoger los excrementos frescos. A menor abundancia de la población se requerirá un mayor número de parcelas. Adicionalmente, se sugiere el empleo simultáneo de cámarastrampa para detectar los sitios de mayor frecuencia de uso de los venados para revisión y obtención de muestras fecales frescas (Bridges y Noss 2011). Estas muestras fecales también pueden servir para otros fines, tales como microhistología con el fin de determinar las especies de plantas en la dieta, así como el análisis de endoparásitos y ciclos hormonales, entre otros (Valdespino et al. 2007; Darimont et al. 2008).

\section{Agradecimientos}

Agradezco a L. A. Escobedo-Morales y T. Pérez-Pérez por su ayuda en la toma de muestras en campo y el análisis en laboratorio, respectivamente; a L. E. Martínez-Romero del área natural protegida Flor del Bosque, por permitirnos el acceso a los venados de ese sitio; al Jardín Botánico Francisco Javier Clavijero del Instituto de Ecología A. C por permitirnos 
realizar el experimento; y a A. R. Trigos-Landa de Laboratorio de Alta Tecnología de Xalapa S. C. por su apoyo técnico para procesar los análisis químicos. Este trabajo forma parte del proyecto CONACYT CB-2009-01-130702. La Red de Biología y Conservación de Vertebrados del Instituto de Ecología A. C. brindó apoyo adicional.

AOAC. 1999. Official methods of analysis of the Association of Official Agricultural Chemists. 16th ed. Association of Official Agricultural Chemists. Gaithersburg, EE.UU.

Aldezabal A., I. Garin, y R. Garcia-Gonzalez. 1993. Concentración de nitrógeno fecal en ungulados estivantes en los pastos supraforestales del parque nacional de Ordesa y Monte Perdido. Revista Pastos 23:101-114.

Bridges A. S., Y A. J. Noss. 2011. Behavior and activity patterns. Pp. 57-69 en Camera Traps in Animal Ecology: Methods and Analyses (O'Connell, A. F., J. D. Nichols, y K. U. Karanth, eds). Springer, EE.UU.

Camargo-Sanabria, A. A., y S. Mandujano. 2011. Comparison of pellet-group counting methods to estimate population density of white-tailed deer in a Mexican tropical dry forest. Tropical Conservation Science 4:230-243.

Darimont, C. T., T. E. Reimchen, H. M. Bryan, y P. C. Paquet. 2008. Faecal-centric approaches to wildlife ecology and conservation; methods, data and ethics. Wildlife Biology in Practices 4:73-87.

Dalgaard, P. 2008. Introduction statistics with R. Springer, EE.UU.

DelGiudice, G. D., P. R. Krausman, E. S. Bellantoni, M. C. Wallace, R. C. Etchberger, y U. S. Seal. 1990. Blood and urinary profiles of free-ranging desert mule deer in Arizona. Journal of Wildlife Disease 26:83-9.

Gallina, S., G. Sánchez-Rojas, A. Buenrostro-Silva, y C. A. López-González. 2014. Comparison of faecal nitrogen concentration between sexes of white-tailed deer in a tropical dry forest in southern Mexico. Ethology Ecology and Evolution DOI: 10.1080/03949370.2014.891537

Gallina, S., S. Mandujano, J. Bello, H. F. López-Arévalo, y M. Weber. 2010. White-tailed deer Odocoileus virginianus (Zimmermann 1780). Pp. 101-118 en Neotropical Cervidology (Duarte, J. M. B. y S. Gonzalez, eds.), IUCN/FUNEP. Gland, Suiza.

Garin, I., A. Aldezabal, R. García-González, y J. R. Aihartza. 2001. Composición y calidad de la dieta del ciervo (Cervus elaphus L.) en el norte de la península Ibérica. Animal Biodiversity Conservation 24:53-63.

Hewitt, D. G. 2011. Nutrition. Pp. 75-105 en Biology and Management of White-tailed Deer, (Hewitt, D., ed.). Taylor and Francis Group. Boca Raton, EE.UU.

Howery, L. D., y J. A. Pfister. 1990. Dietary and fecal concentrations of nitrogen and phosphorus in penned white-tailed deer does. Journal of Wildlife Management 54:383-389.

IrWin, L. L., J. G. Cook, D. E. McWhirter, S. G. Smith, y E. B. Arnett. 1993. Assessing winter dietary quality in bighorn sheep via fecal nitrogen. Journal of Wildlife Management 57:413-421.

Jenks, J. A., R. B. Soper, R. L. Lochmiller, y D. M. Leslie JR. 1990. Effect of exposure on nitrogen and fiber characteristics of white-tailed deer feces. Journal of Wildlife Management 
54:389-391.

Kamler, J., Y M. HomolкA. 2005. Faecal nitrogen: a potential indicator of red and roe deer diet quality in forest habitats. Folia Zoologica 54:89-98.

LesLie JR., D. M., Y E. E. Starkey. 1985. Fecal indices to dietary quality of cervids in old-growth forests. Journal of Wildlife Management 49:142-146.

Leslie JR., D. M., J. A. Jenks, M. Chilelli, y G. R. Lavigne. 1989. Nitrogen and diaminopimelic acid in deer and moose feces. Journal of Wildlife Management 53:216-218.

Mandujano, S. 2010. Potencial del bosque tropical seco para producción de venado cola blanca (Odocoileus virginianus) en México. Pp. 101-130 en Conservación y manejo de fauna cinegética de México Vol. II, (Villarreal Espino Barros, O. A., J. E. Hernández, J. C. Camacho, y F. J. Franco, eds.). Benemérita Universidad Autónoma de Puebla. Puebla, México.

Martínez-Romero, L. E. 2004. Determinación de fechas de aprovechamiento del venado cola blanca (Odocoileus virginianus) a través de hormonas sexuales y comportamiento. Tesis de Maestría, Instituto de Ecología, A. C. Xalapa, México.

Massey, B. N., F. W. Weckerly, C. E. Vaughn, y D. R. McCullough. 1994. Correlations between fecal nitrogen and diet composition in free-ranging black-tailed deer. Southwestern Naturalist 39:165-170.

Ortega-S., A., S. Mandujano, J. Villarreal, M. I. Dimari, H. F. López-Arévalo, M. Correa, Y M. Mouina. 2011. White-tailed deer in Latin America. Pp. 565-597 in Biology and Management of White-tailed Deer, (Hewitt, D., ed.). Taylor and Francis Group. Boca Raton, EE.UU.

Osborn, R. G., y J. A. Jenks. 1998. Assessing dietary quality of white-tailed deer using fecal indices: effects of supplemental feeding and area. Journal of Mammalogy 79:437447.

Osborn, R. G., y T. F. Ginnett. 2001. Fecal nitrogen and 2, 6-diaminopimelic acid as indices to dietary nitrogen in white-tailed deer. Wildlife Society Bulletin 29:1131-1139.

R Development Core Team. 2012. R: a language and environment for statistical computing. Version 2.15.1. http://cran.R-project.org. R Foundation for Statistical Computing. Vienna, Austria.

Robisns, C. T. 1993. Wildlife Feeding and Nutrition. Academic Press. San Diego, EE.UU.

Sacconi, F., M. P. Ponzetta, Y F. Cervasio. 2007. Fecal nitrogen and dietary quality relationships in Fallow deer. Italian Journal of Animal Science 6:862-864.

Thompson, H. N. 1987. Faecal indices to dietary quality: a critique. Journal of Wildlife Management 51: 317-320.

Ueno, M., C. Nishimura, H. Takahashi, K. Kaj, y T. Saitoh. 2007. Fecal nitrogen as an index of dietary nitrogen in two sika deer Cervus nippon populations. Acta Theriologica 52:119-128.

Valdespino, C., y L. E. Martínez-Romero. 2014. Determinación del ciclo reproductivo a partir de grupos fecales. Pp. 87-98 en Monitoreo y manejo del venado cola blanca: Conceptos y métodos (Gallina, S., S. Mandujano, y O. A. Villarreal Espino-Barros, eds.). Instituto de Ecología, A. C. y Benemérita Universidad Autónoma de Puebla. Xalapa, México.

Valdespino, C., R. Martínez-Mota, L. M. García-Feria, y L. E. Martínez-Romero. 2007. Evaluación de eventos reproductivos y estrés fisiológico en vertebrados silvestres a partir de 
sus excretas: evolución de una metodología no invasiva. Acta Zoológica Mexicana 23:151-180.

Verheyden, H., L. Aubry, J. Merlet, P. Petibon, B. Chauveau-Duriot, N. Guillon, y P. Duncan. 2011. Faecal nitrogen, an index of diet quality in roe deer Capreolus capreolus? Wildlife Biology 17:166-175.

Sometido: 17 de septiembre de 2013

Revisado: 4 de mayo de 2014

Aceptado: 14 de octubre de 2014

Editor asociado: Jesús Maldonado 